\title{
Modulation of Decidual Macrophage Polarization by Macrophage Colony-Stimulating Factor Derived from First-Trimester Decidual Cells
}

\section{Implication in Preeclampsia}

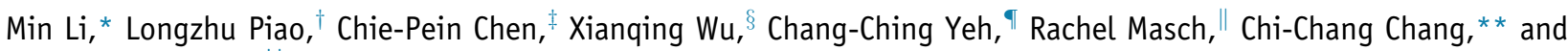

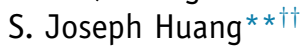

From the Department of Pediatrics, * Yale University School of Medicine, New Haven, Connecticut; the Department of Otolaryngology-Head and Neck Surgery, ${ }^{\dagger}$ The Ohio State University College of Medicine, Columbus, Ohio; the Department of Obstetrics and Gynecology, ${ }^{\ddagger}$ Mackay Memorial Hospital, Taipei, Taiwan; the Department of Obstetrics and Gynecology, ${ }^{\S}$ The Second Xiangya Hospital, Central South University, Changsha, China; the Department of Obstetrics and Gynecology, "Taipei Veterans General Hospital, Taipei, Taiwan; the Department of Obstetrics and Gynecology," Beth Israel Medical Center, New York, New York; the Department of Obstetrics and Gynecology,** E-Da Hospital, I-Shou University, Kaohsiung, Taiwan; and the Department of Obstetrics and Gynecology, ${ }^{\dagger \dagger}$ University of South Florida, College of Medicine, Tampa, Florida

\author{
Accepted for publication \\ December 14, 2015. \\ Address correspondence to $\mathrm{S}$. \\ Joseph Huang, M.D., Ph.D., \\ Department of Obstetrics and \\ Gynecology, E-Da Hospital, \\ I-Shou University, 1 Yi-Da Rd., \\ Jiao-Su Village, Yan-Chao \\ District, Kaohsiung 824, \\ Taiwan. E-mail: jhuang3@ \\ health.usf.edu.
}

\begin{abstract}
During human pregnancy, immune tolerance of the fetal semiallograft occurs in the presence of abundant maternal leukocytes. At the implantation site, macrophages comprise approximately $20 \%$ of the leukocyte population and act as primary mediators of tissue remodeling. Decidual macrophages display a balance between anti-inflammatory and proinflammatory phenotypes. However, a shift to an M1 subtype is reported in preeclampsia. Granulocyte-macrophage colony-stimulating-factor (GM-CSF) and macrophage colonystimulating factor (M-CSF) are major differentiating factors that mediate M1 and M2 polarization, respectively. Previously, we observed the following: i) the preeclamptic decidua contains an excess of both macrophages and GM-CSF, ii) the preeclampsia-associated proinflammatory cytokines, IL-1 $\beta$ and tumor necrosis factor- $\alpha$, markedly enhance GM-CSF and M-CSF expression in cultured leukocyte-free first-trimester decidual cells (FTDCs), iii) FTDC-secreted GM-CSF polarizes macrophages toward an M1 subtype. The microenvironment is a key determinant of macrophage phenotype. Thus, we examined proinflammatory stimulation of FTDC-secreted M-CSF and its role in macrophage development. Immunofluorescence staining demonstrated elevated M-CSF-positive decidual cell numbers in preeclamptic decidua. In FTDCs, IL-1 $\beta$ and tumor necrosis factor- $\alpha$ signal through the NF- $\kappa B$ pathway to induce M-CSF production, which does the following: i) enhances differentiation of and elevates CD163 expression in macrophages, ii) increases macrophage phagocytic capacity, and iii) inhibits signal-regulatory protein $\alpha$ expression by macrophages. These findings suggest that FTDC-secreted M-CSF modulates the decidual immune balance by inducing M2 macrophage polarization and phagocytic capacity in response to proinflammatory stimuli. (Am J Pathol 2016, 186: 1258-1266; http://dx.doi.org/10.1016/j.ajpath.2015.12.021)
\end{abstract}

During human implantation, blastocyst-derived semiallogeneic extravillous trophoblasts (EVTs) invade the underlying decidua and inner third of the myometrium, where they interact with most resident decidual cells and a maternal immune cell population dominated by decidual natural killer cells and macrophages. Invading EVTs surround, breach, and transform uterine spiral arteries into high-capacitance vessels accompanied by enhanced expression of key angiogenic factors and microvascular angiogenesis. ${ }^{1}$ The resulting increased uterine blood flow to the intervillous space promotes

Supported by the National Institute of Child Health and Human Development NIH grant R01HD056123 (S.J.H.).

M.L. and L.P. contributed equally to this work.

Disclosures: None declared. 
development of the fetal-placental unit. ${ }^{2,3}$ In normal human pregnancies, maternal decidual immune cells are exposed to, but do not attack, semiallogeneic fetal antigens. Macrophages comprise approximately $20 \%$ of $\mathrm{CD} 45^{+}$leukocytes, equivalent to $10 \%$ of total cells in the decidua, where they contribute to decidual remodeling at the maternal-fetal interface. Members of the monocytemacrophage lineage display great plasticity. The development of decidual macrophages depends primarily on the local microenvironment. Herein, granulocyte-macrophage colony-stimulating factor (GM-CSF) and/or interferon- $\gamma$ and lipopolysaccharide polarize macrophages toward an M1 phenotype characterized by expression of CD80, CD86, and proinflammatory cytokines, whereas a shift to an M2 phenotype expressing CD163, CD206, and CD209 is induced by macrophage colony-stimulating factor (M-CSF) and/or IL-1 $\beta$, IL-4, or IL-13. The macrophage phenotype is associated with functional changes with M1 macrophages mainly responsible for defense against pathogens, whereas M2 macrophages participate in inhibition of inflammation, debris scavenging, and angiogenesis.

Diverse cell types secrete colony-stimulating factors (CSFs), which then act as potent inducers of macrophage proliferation, differentiation, and activation. ${ }^{4}$ In pregnant women, elevated M-CSF serum levels ${ }^{5}$ rapidly return to baseline after delivery of the fetus. ${ }^{6}$ Macrophages undergo dynamic phenotypic changes that accompany changes in the microenvironment. In the human decidua, macrophages interact primarily with decidual cells, which comprise $>50 \%$ of the total decidual cell population. In early pregnancy, imbalanced decidual macrophage polarization can impair trophoblast function and placentation, and lead to adverse pregnancy outcomes, including preeclampsia, intrauterine fetal growth restriction, miscarriage, and preterm birth. ${ }^{7}$ Our previous studies revealed the following: i) the preeclamptic decidua contained excess macrophages ${ }^{8}$ and elevated GM-CSF levls, ${ }^{9}$ ii) the preeclampsia-associated proinflammatory cytokines, IL-1 $\beta$ and tumor necrosis factor- $\alpha$ (TNF- $\alpha$ ), markedly enhanced GM-CSF and M-CSF expression in cultured leukocyte-free first-trimester decidual cells (FTDCs), ${ }^{10}$ iii) FTDC-secreted GM-CSF polarized macrophages toward an M1 subtype ${ }^{10}$ On the basis of these observations, we hypothesize that FTDC-secreted M-CSF also plays a regulatory role in determining the decidual immune balance by polarizing macrophages toward an M2 subtype in response to proinflammatory stimuli.

In early human pregnancies, local uterine natural killer cells and macrophages are implicated in initiating apoptosis of vascular smooth muscle cells and endothelial cells during decidual vascular remodeling, ${ }^{11,12}$ followed by phagocytosis of apoptotic cells by macrophages. In contrast to the documented loss of endothelial and vascular smooth muscle cells during normal vascular remodeling in the early decidua, loss of villous trophoblast has been observed in several abnormal pregnant conditions, including early pregnancy loss, ${ }^{13}$ preeclampsia, ${ }^{14,15}$ intrauterine fetal growth restriction, ${ }^{16}$ and gestational trophoblast disease. ${ }^{17}$ In addition to its role in regulating tissue homeostasis during pregnancy, phagocytosis of apoptotic cells by decidual macrophages also serves as a key protective mechanism against aberrant inflammation secondary to release of cellular contents while inducing regulatory T-cell development and/or T-cell anergy as well as enhanced secretion of type 2 helper T-cell cytokines that promote maternal immune tolerance of the fetal semiallograft. Paradoxically, macrophages have also been shown to phagocytose viable cells by a process termed phagoptosis. ${ }^{18}$ Our previous results demonstrated that proinflammatory stimulus-activated FTDCs enhanced the activity of macrophages in inducing apoptosis of first-trimester EVTs. ${ }^{10}$ These observations suggest that a balance between scavenger and killing activities of macrophages is crucial in maintaining decidual homeostasis during early human pregnancy.

Discrimination between healthy and unwanted cells is critical in maintaining cellular homeostasis and tissue remodeling at the maternal-fetal interface. Dysregulation of this balance can elicit tissue damage because of either excess removal of viable cells or insufficient removal of unwanted/compromised cells. A Don't-Eat-Me signal generated by binding of signal-regulatory protein $\alpha$ (SIRP $\alpha)$ expressed on the surface of macrophages and its ubiquitously expressed ligand, CD47, is a critical regulator of such phagocytic activity. ${ }^{19}$ Thus, the current study initially demonstrated the presence of M-CSF in preeclamptic decidua by immunofluorescence staining. The signaling pathways mediating M-CSF-, IL-1 $\beta-$, or TNF- $\alpha$-enhanced expression by FTDCs were then examined. Subsequently, the functional role of M-CSF secreted by IL- $1 \beta-$ or TNF- $\alpha-$ stimulated FTDCs in affecting the decidual immune balance by regulating macrophage polarization and phagocytic capacity was evaluated, followed by assessing the expression of SIRP $\alpha$ on macrophages. The results obtained from these studies are expected to shed light on the pathogenesis of adverse pregnancy outcomes.

\section{Materials and Methods}

\section{Immunofluorescence Staining}

Decidua basalis was obtained from patients with preeclampsia under Institutional Review Board approval at Mackay Memorial Hospital (Taipei, Taiwan). Preeclampsia was defined as the occurrence of elevated blood pressure $(\geq 140 / 90$ $\mathrm{mm} \mathrm{Hg}$ ) and proteinuria ( $>1+$ on a urine dip stick) identified on two occasions, 6 hours apart after 20 weeks of gestation in the absence of signs and symptoms of chorioamnionitis or chronic villitis. All cases (37 to 40 weeks) were delivered by cesarean section without labor. Gestational age (GA)matched controls without labor and signs of preeclampsia were also obtained after cesarean delivery. Serial sections $(8 \mu \mathrm{m}$ thick) of OCT-embedded decidua were stained with anti-human M-CSF in Tris-buffered saline (R\&D Systems, Minneapolis, $\mathrm{MN}$ ) for 1 hour at $25^{\circ} \mathrm{C}$. Immunostaining 
specificity was assessed by substituting a mouse monoclonal IgG isotype ( $R \& D$ Systems) for the primary antibody. The sections were then incubated with fluorescein isothiocyanate-conjugated secondary antibodies for 1 hour. After washing, the sections were incubated with goat antihuman vimentin or cytokeratin 7 antibody for 1 hour at $25^{\circ} \mathrm{C}$, followed by incubation with rhodamine-conjugated rabbit anti-goat antibody (1:400; Sigma-Aldrich, St. Louis, MO) or goat anti-rabbit antibody (1:100; Millipore, Temecula, CA) for 1 hour. Finally, the sections were washed and stained with DAPI (Sigma-Aldrich), and mounted in a nonfade mounting medium (Life Sciences International, Basingstoke, UK). Immunofluorescence was examined under a Zeiss microscope (Müchen-Hallbergmoos, Germany) equipped with a cooled charge-coupled device camera (Axiocam HRm; Zeiss).

\section{Cell Isolation and Culture}

FTDCs were isolated and cultured, as previously described. ${ }^{11}$ Briefly, decidual specimens from elective terminations between 6 and 12 weeks of gestation were obtained under Institutional Review Board approval at Beth Israel Medical Center (New York, NY) and The Ohio State University (Columbus, $\mathrm{OH}$ ). Tissues were minced and digested with $0.1 \%$ collagenase type IV, $0.01 \%$ DNase in Ham's F- 10 . The digestate was purified on 60\%/50\%/40\% Ficoll-Paque Plus (GE Health Care, Piscataway, NJ). Decidual cells were collected from the $40 \%$ layer and cultured in basal medium (phenol red-free 1:1 v/v mix of Dulbecco's modified Eagle's medium and Ham's F-12; Sigma-Aldrich) supplemented with $100 \mathrm{U} / \mathrm{mL}$ penicillin, $100 \mu \mathrm{g} / \mathrm{mL}$ streptomycin, $0.25 \mathrm{ng} / \mathrm{mL}$ fungizone (Invitrogen, Carlsbad, CA), and 10\% charcoal-stripped calf serum (Gemini Bio-Products, West Sacramento, CA). The cells were passaged until free of immune cell contamination, as determined by flow cytometric analysis of anti-CD14 and anti-CD45 (BD Pharmingen, San Diego, CA). Leukocyte-free confluent decidual cells were primed with $10^{-8} \mathrm{~mol} / \mathrm{L}$ estradiol plus $10^{-7} \mathrm{~mol} / \mathrm{L}$ medroxyprogesterone acetate for 7 days. Decidualization was confirmed by enhanced expression of prolactin and plasminogen activator inhibitor-1 and inhibited expression of interstitial collagenase and stromelysin-1 (data not shown). These cells were then cultured in serum-free basal medium with or without $1 \mathrm{ng} / \mathrm{mL} \mathrm{IL}-1 \beta$ or TNF- $\alpha$ (R\&D Systems) for 24 hours. Conditioned medium (CM) supernatants and whole cell lysates or total RNA were collected and stored at $-80^{\circ} \mathrm{C}$ for future use.

\section{Signaling Pathway Mediation of M-CSF Production in FTDCs}

FTDCs were preincubated with $20 \mu \mathrm{mol} / \mathrm{L}$ mitogen-activated protein kinase (MAPK) inhibitor (PD980589), $20 \mu \mathrm{mol} / \mathrm{L}$ Jun kinase inhibitor (SP600125), $1 \mu \mathrm{mol} / \mathrm{L}$ p38 kinase inhibitor (SB203580; Sigma-Aldrich), or $5 \mu \mathrm{mol} / \mathrm{L}$ NF- $\kappa B$ inhibitor (BAY11-7082; Invitrogen) for 1.5 hours before incubation with $1 \mathrm{ng} / \mathrm{mL}$ IL- $1 \beta$ or TNF- $\alpha$. CM was collected after 24 hours for measurements of M-CSF using a commercial enzyme-linked immunosorbent assay (ELISA) kit from eBioscience (San Diego, CA), according to the manufacturer's instructions. Total cell protein levels were measured by a modified Lowry assay (Bio-Rad Labs Inc., Hercules, CA).

\section{ELISA}

\section{M-CSF Expression in Decidual Tissue}

Frozen tissues were homogenized in RIPA lysis buffer (2\% NP-40, $0.02 \%$ SDS, $1 \%$ sodium deoxycholate, and $1 \times$ phosphate-buffered saline) with protease inhibitor cocktail (Sigma-Aldrich). Total protein levels were measured using protein assay dye reagent (PAK500; Strong Biotech, Taipei, Taiwan). M-CSF levels were measured using an ELISA kit by following the manufacturer's instruction (R\&D Systems). The sensitivity range was 1.74 to $47.3 \mathrm{pg} / \mathrm{mL}$. The intra-assay and interassay CVs were $2.1 \%$ to $7.8 \%$ and $4.5 \%$ to $12.7 \%$, respectively.

\section{SIRP $\alpha$ Expression in Macrophages}

Total cell protein levels were measured by a modified Lowry assay (Bio-Rad Labs Inc.). Commercial ELISA kits measured immunoreactive levels of SIRP $\alpha$ in macrophage cell lysates, according to the manufacturer's instructions (AntibodiesOnline Inc., Atlanta, GA). The sensitivity of the assay was $61 \mathrm{pg} / \mathrm{mL}$. The intra-assay and interassay CVs were $10 \%$ and $12 \%$, respectively.

\section{Macrophage Differentiation}

Peripheral blood mononuclear cells were isolated from the interphase after Ficoll-Paque centrifugation of $100 \mathrm{~mL}$ peripheral blood from healthy reproductive age female donors under Institutional Review Board approval at The Ohio State University. Monocytes were purified with anti-CD14 paramagnetic beads (Miltenyi Biotec, Auburn, CA), according to the manufacturer's instruction. The purity of monocytes $(>95 \%)$ was determined by flow cytometric analysis. $\mathrm{CD} 14^{+}$monocytes $\left(5 \times 10^{5}\right.$ cells $\left./ \mathrm{mL}\right)$ were cultured with $\mathrm{CM}$ from unstimulated or IL- $1 \beta$ - or TNF- $\alpha$-treated FTDCs with or without excess anti-M-CSF neutralizing antibody (R\&D Systems) for 7 days. Before use, the $\mathrm{CM}$ was incubated with an excess of either anti-IL-1 $\beta$ or anti-TNF- $\alpha$ neutralizing antibody (R\&D Systems) to eliminate residual cytokines. Then, macrophages were harvested and blocked with normal human IgG followed by staining with anti-human CD11B-PE, CD163-APC, and CD80PECy5 (R\&D Systems), according to the manufacturer's instruction. The cells were analyzed by a BD LSRII flow cytometer using FACSDiva software version 7 (BD Biosciences, San Jose, CA).

\section{Phagocytosis Assay}

A human first trimester EVT cell line, HTR-8/SVNeo cells, were first labeled with PKH67 (Sigma-Aldrich). Apoptosis 

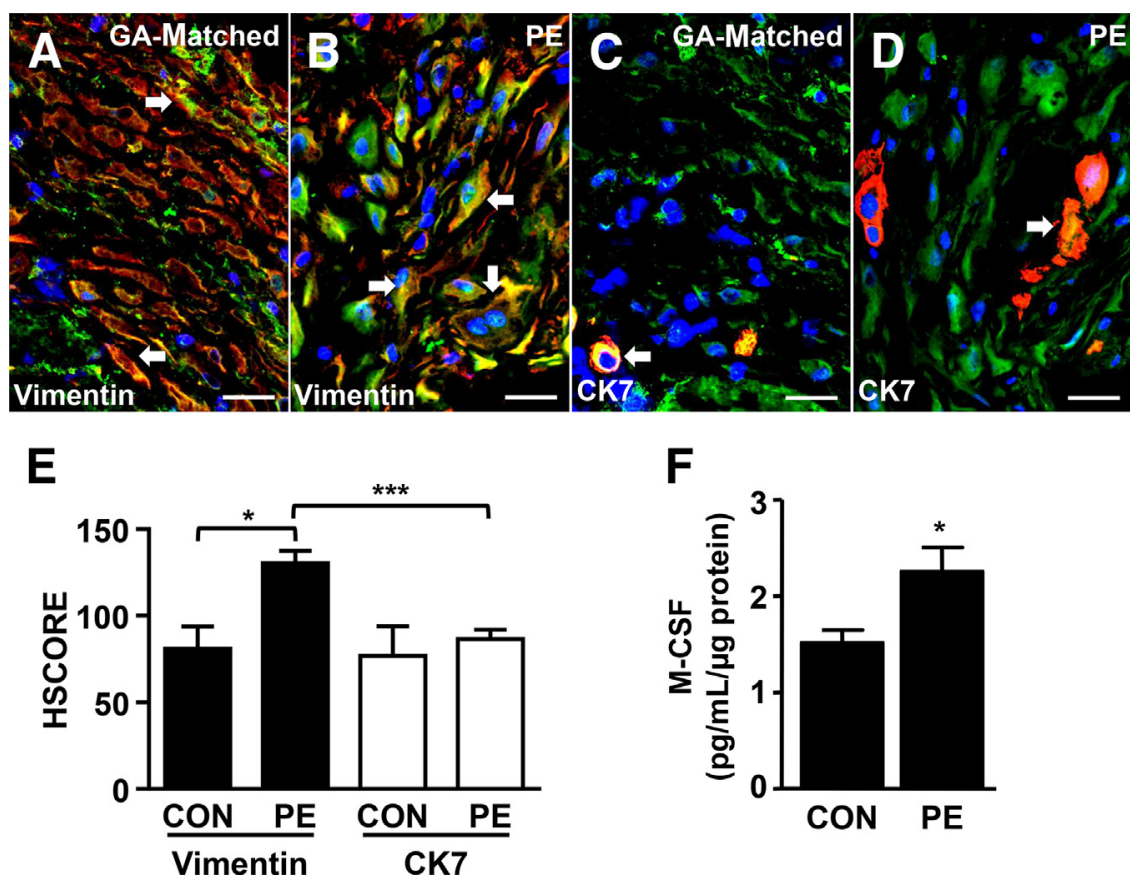

$\mathbf{F}$

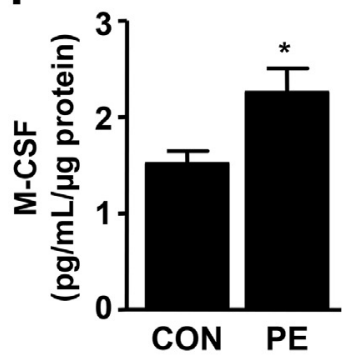

Figure 1 Macrophage colony-stimulating factor (M-CSF) expression is increased in decidual cells from women with preeclampsia. Representative M-CSF immunofluorescence staining of the decidual cell marker, vimentin (red), in gestational age (GA)-matched (A) and preeclamptic (PE; B) decidua obtained from serial frozen sections of placental bed biopsy specimens. Serial sections from corresponding tissue were also immunostained for the extravillous trophoblast marker, cytokeratin (CK) 7 (red), using GA-matched (C) and PE decidua (D). M-CSF expression in the decidua was assessed by immunofluorescence staining (green). Arrows indicate cells double stained with either vimentin or CK7 with M-CSF. E: HSCORE semiquantifies the M-CSF immunoreactivity. F: Tissue enzyme-linked immunosorbent assay measured M-CSF expression in placental bed biopsy specimens from GA-matched and PE patients. Results are expressed as means \pm SEM (E and $\mathbf{F}) . n=11$ (E and $\mathbf{F}) .{ }^{*} p<0.05$, $\star * * P<0.005$. Scale bar $=50 \mu \mathrm{m}(\mathbf{A}-\mathbf{D})$. Original magnification, $\times 400($ A-D). CON, control. of PKH67-labeled EVTs was induced by serum depletion. Apoptotic bodies of PKH67-labeled EVTs were added to macrophages treated with CM from FTDC cultures with or without M-CSF neutralizing antibody (R\&D Systems) at a ratio of 1:3 for 6 hours. After incubation, cells were pelleted and stained with anti-CD11b-PE. Phagocytosis was evaluated by gating cells with double staining of PKH67 (green) and CD11b-PE (red) with BD LSRII flow cytometer using BD FACSDiva software version 7 . The phagocytic index was calculated as the percentage of phagocytosing $\mathrm{CD} 11 \mathrm{~b}^{+}$ macrophages $\times$ mean green fluorescence.

\section{Quantitative RT-PCR}

Total RNA was extracted from macrophages using a total RNA purification plus kit (Norgen Biotek Corp., Thorold, ON, Canada). Reverse transcription used SuperScriptIII First-Strand Synthesis System from Invitrogen. The quantitative PCR with SYBR Green-based detection was performed using the following primer sets: $\beta$-actin, 5'-CGTACCACTGGCATCGTGAT-3' (forward) and 5'-GTGTTGGCGTACAGGTCTTTG-3' (reverse); and SIRP $\alpha, 5^{\prime}$-TGCCAGAGAAATAACACAGGACA-3' (forward) and 5'-CTGGCATACTCCGTG-3' (reverse) (Integrated DNA Technologies, Coralville, IA). Product sizes of $\beta$-actin and SIRP $\alpha$ were 452 and $103 \mathrm{bp}$, respectively. Quantitation of unknowns was determined and adjusted to quantitative expression of $\beta$-actin in specific samples. Melting curve analysis determined the specificity of the amplified products and the absence of primer-dimer formation.

\section{Statistical Analysis}

The variance and normality of data from HSCORE, tissue ELISA, M-CSF, SIRP $\alpha$ ELISAs, phagocytosis assays, macrophage differentiation, and quantitative RT-PCR were first examined. Then, the statistical significance of results with equal variance was examined by $t$-test, assuming equal variance. The results with unequal variance that either passed or failed the normality test were then evaluated by $t$-test, assuming unequal variance or the Mann-Whitney rank sum test, respectively. $P<0.05$ was considered significant.

\section{Results}

M-CSF Expression Is Increased in Decidua from Women with Preeclampsia

Decidual cells (Figure 1, A and B) and EVTs (Figure 1, C and D) were identified by vimentin and cytokeratin 7 staining (red), respectively, in OCT-embedded serial sections from GA-matched control and preeclamptic decidua. Coimmunostaining with M-CSF (green) showed that M-CSF was mainly expressed in vimentin-positive decidual cells (orange to yellow) with significantly higher expression observed in preeclamptic decidua than in GA-matched control specimens (Figure 1E). Unlike decidual cells, M-CSF expression in EVTs did not differ between GA-matched control and preeclamptic decidua. Consistent with the immunostaining results, tissue ELISA measurements indicated that extracts of decidua from women with preeclampsia contained significantly higher levels of immunoreactive M-CSF compared with GA-matched control decidua (Figure 1F).

Decidual Cell-Derived M-CSF Regulates Differentiation and M2 Polarization of Macrophages

Figure 2 displays the effects of FTDC-derived M-CSF on macrophage development. Morphologically, CM from 


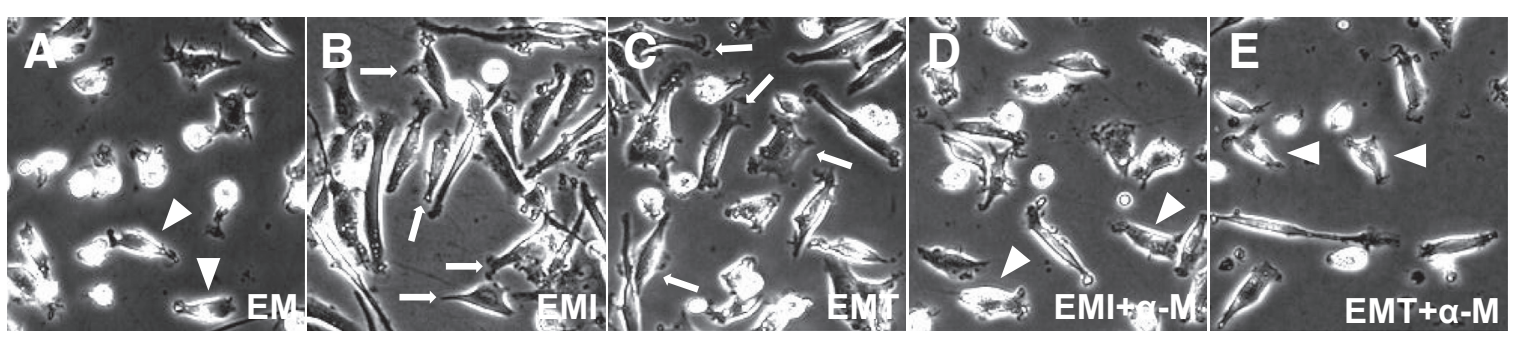

$\mathbf{F}$

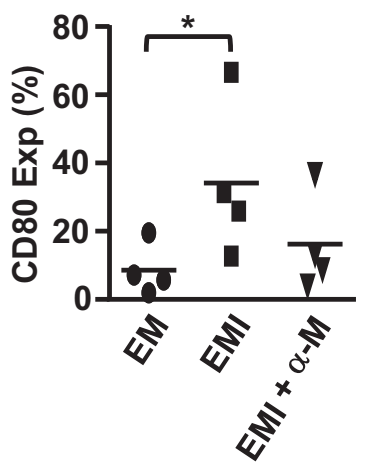

G

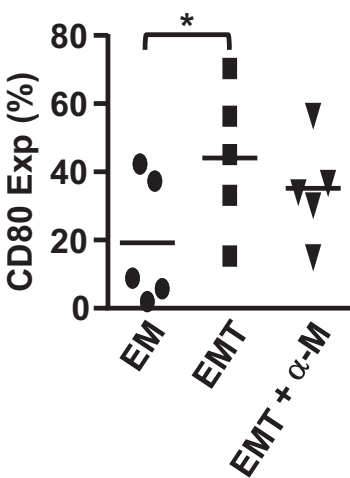

H

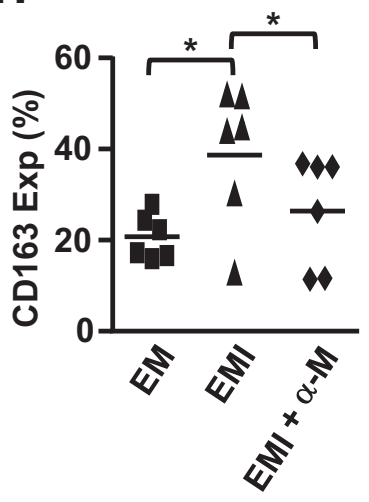

I

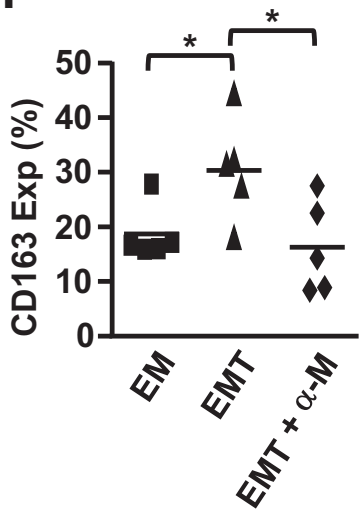

Figure 2 Macrophage differentiation is enhanced by macrophage colony-stimulating factor (M-CSF) produced by IL-1 $\beta$ - or tumor necrosis factor (TNF)$\alpha$-treated first-trimester decidual cells (FTDCS). Peripheral monocytes were differentiated in conditioned medium [from FTDCs treated with control (EM; A), IL-1 $\beta$ (EMI; B) or TNF- $\alpha$ (EMT; C)] for 7 days with or without preincubation with an anti-human M-CSF antibody (D and E). Flow cytometric analysis assessed the expression of CD80 (F and $\mathbf{G})$ and $C D 163$ on $\mathrm{CD}_{11 b^{+}}(\mathbf{H}$ and $\mathbf{I})$ macrophages. Arrows indicate cells with increased ruffles and pseudopods. Arrowheads indicate cells with fewer ruffles and pseudopods. Results are expressed as means \pm SEM $(\mathbf{F}-\mathbf{I}) . n=11(\mathbf{F}-\mathbf{I}) .{ }^{*} P<0.05$. Original magnification, $\times 400(\mathbf{A}-\mathbf{E}) . \alpha-M$, anti-M-CSF; Exp, expression.

IL-1 $\beta$ - (Figure 2B) or TNF- $\alpha$-treated (Figure 2C) FTDCs enhanced macrophage differentiation, as indicated by more prominent membrane ruffles and numbers of pseudopods compared with control CM (Figure 2A). These morphological changes were decreased by preincubation with an anti-M-CSF neutralizing antibody (Figure 2, D and E). Moreover, CD80 (Figure 2, F and G) and CD163 (Figure 2, H and I) expression by macrophages was induced by incubation with $\mathrm{CM}$ from IL- $1 \beta-$ or TNF- $\alpha$-stimulated FTDCs. By comparison, treatment with an anti-M-CSF neutralizing antibody significantly reduced CD163, but not CD80, expression (Figure 2, $\mathrm{H}$ and I).

\section{IL-1 $\beta$ and TNF- $\alpha$ Both Signal through NF- $\kappa$ B Pathway} for M-CSF Production in FTDCs

Consistent with our previous results, ${ }^{10}$ incubation of confluent FTDCs with IL- $1 \beta$ or TNF- $\alpha$ significantly enhanced M-CSF production by 51-fold (Figure 3A) and 52-fold (Figure 3B), respectively. Because MAPK and $\mathrm{NF}-\kappa \mathrm{B}$ pathways are each documented to mediate elevated expression of several chemokines in FTDCs in response to IL-1 $\beta$ or TNF- $\alpha,{ }^{20}$ the current study questioned whether either or both pathways mediate proinflammatoryenhanced M-CSF expression by FTDCs. Therefore, confluent FTDCs were preincubated with an inhibitor of specific signaling molecules of the MAPK or NF- $\kappa \mathrm{B}$ pathways before IL- $1 \beta$ or TNF- $\alpha$ treatment. The NF- $\kappa B$ inhibitor, BAY 11-7082, significantly inhibited M-CSF production induced by either IL-1 $\beta$ (Figure $3 \mathrm{~A}$ ) or TNF- $\alpha$ (Figure 3B) by 3.99- and 2.64-fold, respectively. By contrast, inhibition of M-CSF expression by the Jun kinase inhibitor, SP600125, attained only borderline statistical significance. Moreover, M-CSF levels induced by either IL-1 $\beta$ or TNF- $\alpha$ were not affected by pretreatment with either a MAPK inhibitor (PD980589) or a p38 kinase inhibitor (SB203580).

Enhanced Phagocytic Capacity of Macrophages by IL- $1 \beta$ - or TNF- $\alpha$-Treated FTDCs Is Attenuated by an Anti-M-CSF Neutralizing Antibody

Macrophage function was evaluated by examining the influence of FTDCs on phagocytic activity (Figure 4A). Phagocytosis of EVT-derived apoptotic bodies by macrophages was augmented by incubations with CM from IL-1 $\beta$ (Figure 4, B and F) or TNF- $\alpha$-treated (Figure 4, C and G) FTDCs. Specifically, a greater number of macrophages (red) engulfing apoptotic bodies (green) was observed with a greater number of apoptotic bodies per macrophage. Furthermore, pretreatment with an anti-M-CSF neutralizing antibody significantly reduced the phagocytic capacity of macrophages that was enhanced by incubation with $\mathrm{CM}$ from IL-1 $\beta$ - (Figure 4, D and F) or TNF- $\alpha$-treated (Figure 4, E and G) FTDCs. 


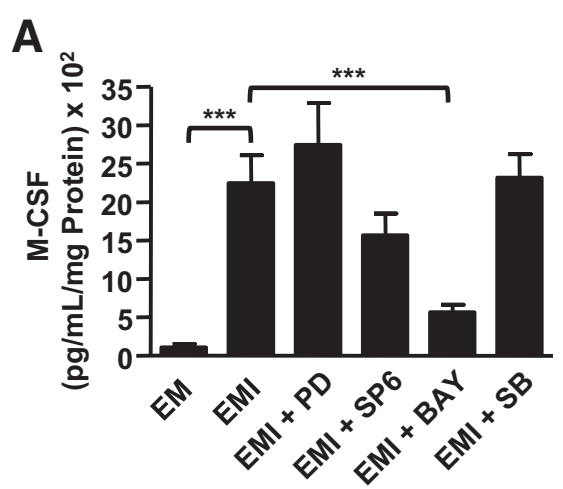

B

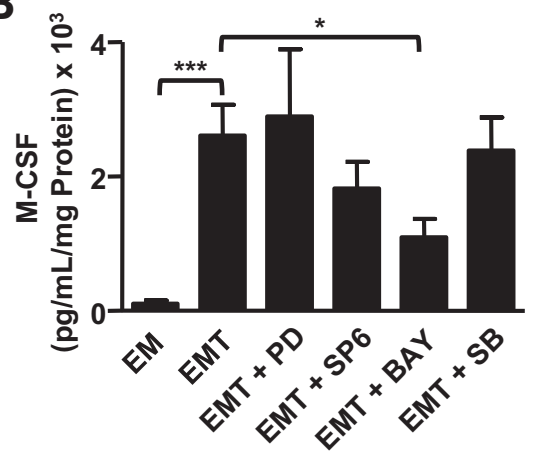

Figure 3 The NF- $\kappa B$ signaling pathway mediates IL-1 $\beta$ - or tumor necrosis factor (TNF)- $\alpha$-induced macrophage colony-stimulating factor (M-CSF) expression by first-trimester decidual cells (FTDCs). FTDCs were preincubated with mitogen-activated protein kinase inhibitor [PD98059 (PD)], Jun kinase inhibitor [SP600125 (SP6)], p38 inhibitor [SB203580 (SB)], or NF- $\kappa B$ inhibitor [BAY11-7082 (BAY)] for 1 hour, followed by stimulation with IL-1 $\beta$ (EMI; A) or TNF- $\alpha$ (EMT; B) for 24 hours. Levels of M-CSF in conditioned medium were measured by enzyme-linked immunosorbent assays. Results are expressed as means \pm SEM (A and B). $n=5$ (A and $\mathbf{B}) .{ }^{*} P<0.05,{ }^{* *} P<0.005$. EM, conditioned medium from control.

\section{SIRP $\alpha$ Expression on Macrophages Is Inhibited by IL- $1 \beta$ - or TNF- $\alpha$-Treated FTDCs}

The binding of SIRP $\alpha$ on macrophages with CD47 on other cell types is crucial in preventing macrophages from phagocytosing both cell debris and vital cells. Thus, SIRP $\alpha$ expression on macrophages was examined to identify a potential mechanism regulating changes in phagocytic capacity of macrophages incubated with $\mathrm{CM}$ from IL- $1 \beta-$ or TNF- $\alpha$-treated FTDCs. Significantly decreased macrophage-expressed SIRP $\alpha$ mRNA levels were observed in response to incubation with $\mathrm{CM}$ from IL- $1 \beta$ - or TNF- $\alpha$-treated FTDCs by 7.29 - and 6.42 fold, respectively (Figure $4 \mathrm{H}$ ). This inhibition was reduced by preincubation with an anti-M-CSF antibody. Consistent with these mRNA results, ELISA measurements indicated that CM from IL- $1 \beta-$ or TNF- $\alpha$-treated FTDCs inhibited SIRP $\alpha$ protein expression by 2.02- and 1.75-fold, respectively (Figure 4I). Although anti-M-CSF pretreatment blocked the inhibitory effects of $\mathrm{CM}$ from IL-1 $\beta$-treated FTDCs on SIRP $\alpha$ protein expression on macrophages by 2.11 -fold, this anti-M-CSF treatment did not affect SIRP $\alpha$ protein expression induced by $\mathrm{CM}$ from TNF- $\alpha$-treated FTDCs.

\section{Discussion}

Increased numbers of decidual macrophages expressing elevated levels of GM-CSF, M-CSF, IL-8, and monocyte chemoattractant protein- ${ }^{9-11}$ were demonstrated in pregnancies complicated by preeclampsia, intrauterine fetal growth restriction, and spontaneous abortion. ${ }^{8,21}$ Decidual macrophages were also found to be aberrantly activated in pregnancies complicated by preeclampsia ${ }^{22}$ and spontaneous abortion. ${ }^{23}$ Impaired EVT invasion of uterine spiral arteries leading to incomplete vascular remodeling and reduced uteroplacental blood flow required by the developing fetalplacental unit is implicated in the later development of preeclampsia. ${ }^{2}$ These events are accompanied by a large number of decidual macrophages, suggesting that preeclampsia is associated with excess macrophage infiltration. However, in addition to recruitment from blood, recent studies suggest that increased in situ development is a key contributor to macrophage accumulation during inflammatory processes, particularly during M2-dominated inflammation. ${ }^{24} \mathrm{Com}$ plementing our previous results, the current observations indicate that an aberrant accumulation of macrophages in the decidua related to preeclampsia or miscarriage may reflect either increased monocyte recruitment ${ }^{8}$ or enhanced development to macrophages induced by FTDC-derived chemokines $^{11}$ and/or various CSFs. ${ }^{11}$

In vitro, macrophages exhibit a potential to develop to various subtypes in response to specific cytokines. ${ }^{25}$ Among these cytokines, M-CSF plus IL-10 induces macrophages that display the closest phenotype to decidual macrophages. Our previous results indicated that M1 polarization is enhanced by incubation with CM derived from FTDCs treated with IL-1 $\beta$ or TNF- $\alpha .{ }^{10}$ Paradoxically, the current study demonstrates that $\mathrm{M} 2$ polarization of macrophages is also increased by M-CSF secreted by FTDCs, after incubation with either of these proinflammatory cytokines. These results suggest the existence of a dynamic change between M1 and M2 polarization during the pathogenesis of preeclampsia. GM-CSF and M-CSF are the main cytokines responsible for $\mathrm{M} 1$ and $\mathrm{M} 2$ macrophage polarization, respectively. ${ }^{26}$ Compared with our previous GM-CSF measurements, ${ }^{9,11}$ the current results indicate that considerably lower M-CSF levels are produced by FTDCs treated with either IL-1 $\beta$ or TNF- $\alpha$. Thus, the M1 subtype induced by FTDC-secreted GM-CSF is likely to be the predominant decidual macrophage subtype in response to proinflammatory stimulation with M-CSF-induced M2 polarization of macrophages serving as a compensatory response. In addition to M1/M2 polarization, differential expression levels of CD11c, CD209, and CD206 are suggested as markers that identify decidual macrophage subtypes in early pregnancy. ${ }^{27}$ Future studies are planned to examine the 


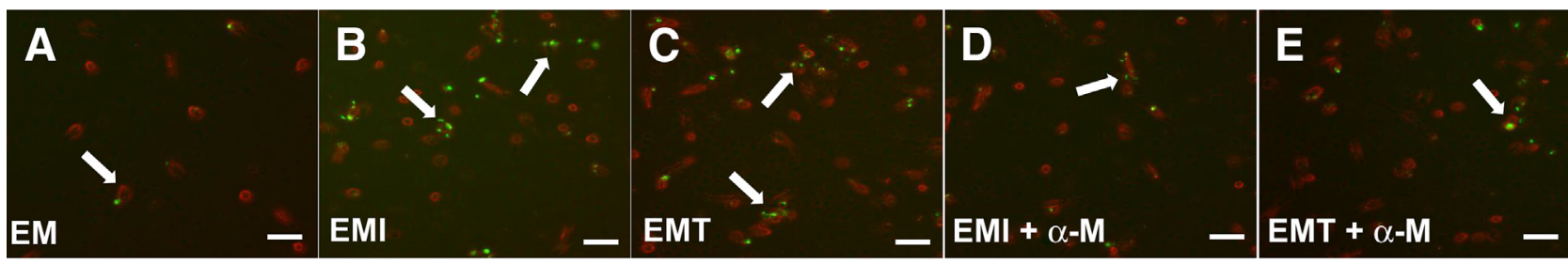

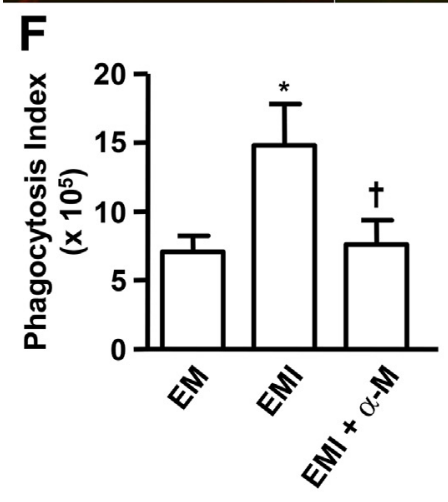

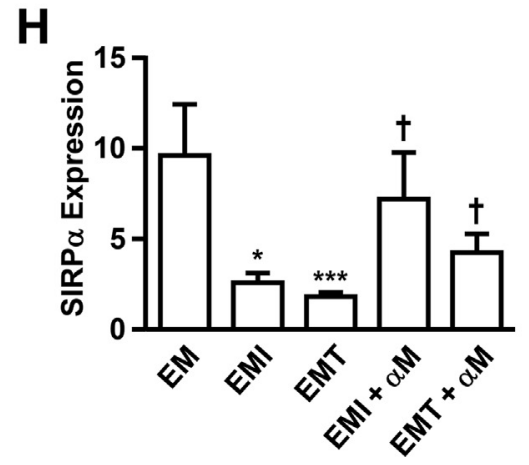

G

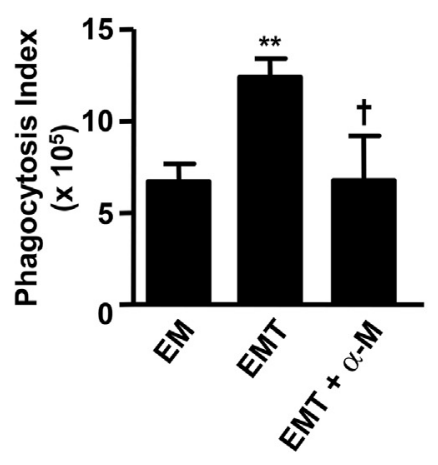

I

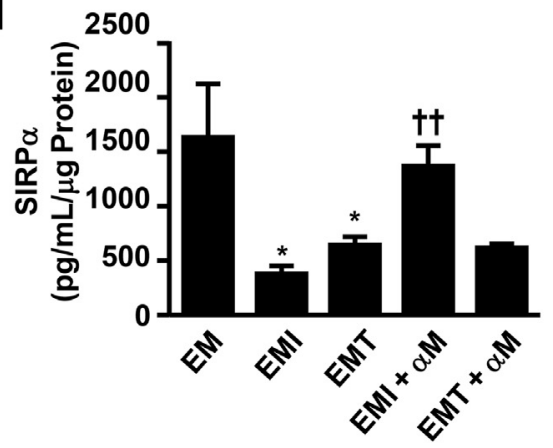

Figure 4 Macrophage colony-stimulating factor (M-CSF) in IL-1 $\beta$ - or tumor necrosis factor (TNF)- $\alpha$-stimulated first-trimester decidual cells (FTDCs) enhances phagocytic capacity of macrophages and down-regulates signal-regulatory protein (SIRP) $\alpha$ expression on macrophages. Macrophages (red) treated with conditioned medium (CM) from control (EM; A), IL-1 $\beta$ (EMI; B), or TNF- $\alpha$ (EMT; C) stimulates FTDCs with or without anti-human M-CSF antibody in the presence of PKH67labeled HTR-derived apoptotic bodies (green; D and E). Macrophages engulfing apoptotic bodies are indicated by arrows. $\mathbf{F}$ and $\mathbf{G}$ : The phagocytosis index was calculated as follows: the percentage of $\mathrm{CD}_{11 \mathrm{~b}^{+}}$phagocytosing macrophages $\times$ mean value of green fluorescence measured by flow cytometry. SIRP $\alpha$ expression on macrophages was assessed by quantitative RT-PCR (H) and enzyme-linked immunosorbent assay (I). Results are expressed as means \pm SEM $(\mathbf{F}-\mathbf{I}) . n=3(\mathbf{F}-\mathbf{I})$. ${ }^{*} P<0.05,{ }^{* *} P<0.01$, and ${ }^{* * *} P<0.005$ versus $\mathrm{EM} ;{ }^{\dagger} P<0.05,{ }^{\dagger \dagger} P<0.01$ versus EMI and EMT. Scale bar $=50 \mu \mathrm{m}(\mathbf{A}-\mathbf{E})$. Original magnification, $\times 200(\mathbf{A}-\mathbf{E}) . \alpha-M$, anti-M-CSF; HTR, HTR-8/SVNeo cells. expression of CD11c, CD209, and CD206 under the influence of $\mathrm{CM}$ derived from proinflammatory cytokine versus control-treated FTDCs. In several pathological conditions, macrophages express a unique or mixed phenotypic repertoire. $^{28,29}$ Therefore, further studies are also planned to elucidate the distribution of decidual macrophage subtypes in various pregnancy complications.

Previous results from our laboratory revealed that IL- $1 \beta$ and TNF- $\alpha$ each signal primarily through NF- $\kappa \mathrm{B}$ and MAPK, but not phosphatidylinositol 3-kinase, to activate the secretion by FTDCs of macrophage/dendritic cell-recruiting chemokines. ${ }^{20}$ However, the signaling pathways that regulate the production of M-CSF in response to IL- $1 \beta$ and TNF- $\alpha$ in FTDCs were unclear. Attempting to clarify this situation, the current study identifies NF- $\kappa B$ signaling as the pathway primarily responsible for M-CSF production induced in FTDCs by IL- $1 \beta$ and TNF- $\alpha$, with Jun kinase perhaps playing a minor role in this regulation. Taking our current and previous studies together, NF- $\kappa \mathrm{B}$ is revealed as the common major signaling pathway mediating both chemokine and M-CSF production in proinflammatory cytokine-stimulated FTDCs. These observations suggest that modulation of NF- $\kappa \mathrm{B}$ signaling can be used as a potential preventative and therapeutic strategy in battling adverse pregnancy outcomes characterized by proinflammatory immune profiles.

Complementing previous studies evaluating the regulation of immune tolerance in the context of the major histocompatibility complex expression and functions of $\mathrm{T}$, $\mathrm{B}$, and natural killer cells during implantation, ${ }^{30}$ increasing attention has now focused on modulation by phagocytes of homeostatic turnover and elimination of self cells. Specifically, in addition to antigen presentation, the phagocytic activity of macrophages and dendritic cells plays major roles in determining the critical immune balance that prevents rejection of the semiallogeneic embryo. In this regard, the current study demonstrates that CM obtained from incubations of FTDCs with either IL$1 \beta$ or TNF- $\alpha$ promotes the phagocytic capacity of macrophages in concert with suppression of SIRP $\alpha$ expression on macrophages. M-CSF is a key mediator of these effects. Previously, SIRP $\alpha$ was identified as an inhibitory receptor expressed on myeloid and neuronal cells that interacts with a ubiquitous membrane protein, CD47. ${ }^{19}$ Binding of SIRP $\alpha$ to $\mathrm{CD} 47$ negatively regulates phagocytosis of host cells by myeloid cells, while initiating 


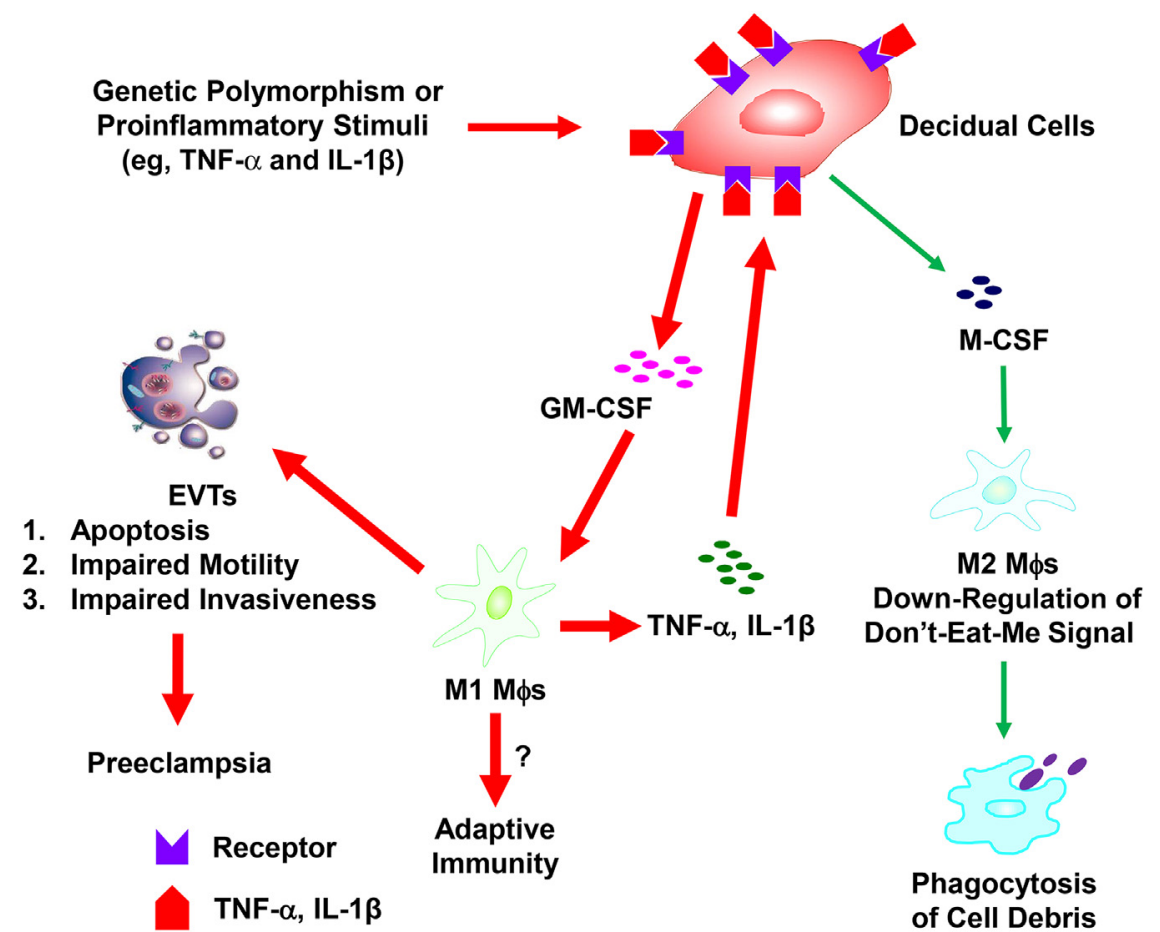

Figure 5 Hypothetical model of the pathogenesis of preeclampsia. The production of both granulocyte-macrophage colony-stimulating factor (GM-CSF) and macrophage colony-stimulating factor (M-CSF) in first-trimester decidual cells (FTDCs) is up-regulated by proinflammatory stimuli. Decidual macrophages $(M \theta)$ polarize to $M 1$ or $M 2$ subtypes by GM-CSF or M-CSF, respectively. The activated M1 macrophages, in turn, secrete more IL-1 $\beta$ and tumor necrosis factor (TNF)- $\alpha$ to generate a feed-forward cycle to FTDCs for more GM-CSF and M-CSF production. M1 macrophages, therefore, induce extravillous trophoblast (EVT) apoptosis and inhibit EVT invasion with the balance of M2 macrophages that phagocytose cell debris of apoptotic EVTs. Red arrows represent the predominant responses in favor of the induction of M1 macrophage polarization and the development of preeclampsia. Green arrows indicate the responses in favor of M2 macrophage polarization. bidirectional signaling through both SIRP $\alpha$ and CD $47 .^{31}$ The key role played by $\mathrm{CD} 47$ in the activation of a Don't-Eat-Me signal taken together with the important contribution of both EVTs and endothelial cells to normal placentation indicates the need for future investigations that scrutinize the influence of FTDCs on the expression of CD47 by both cell types.

In summary, the current study demonstrates the following: i) increased M-CSF expression in the decidua from patients with preeclampsia, ii) the proinflammatory cytokines, IL- $1 \beta$ or TNF- $\alpha$, signal primarily through the $\mathrm{NF}-\kappa \mathrm{B}$ pathway to up-regulate M-CSF expression in FTDCs, leading to M2 polarization of macrophages, and iii) the phagocytic capacity of M2 macrophages is consistently enhanced, possibly via down-regulation of SIRP $\alpha$. These observations are summarized in Figure 5, which indicates that excess levels of TNF- $\alpha$ or IL- $1 \beta$ associated with inflammatory states or maternal genetic polymorphisms target decidual cells to enhance production of macrophage-recruiting chemokines and macrophage-activating M-CSF and GM-CSF. ${ }^{9,32}$ Activated macrophages are a rich source of TNF- $\alpha$ and IL-1 $\beta$. Both proinflammatory cytokines can target decidual cells to promote a feed-forward cycle of chemoattracting macrophages and the production of TNF- $\alpha$, IL- $1 \beta$, and CSFs. Consistent with in vivo observations, ${ }^{33}$ our in vitro studies revealed that apoptosis of extravillous EVTs is induced by M1 macrophages under the influence of TNF- $\alpha-$ or IL$1 \beta$-treated decidual cells. ${ }^{10}$ The current study extends this finding by demonstrating an increased phagocytosis by M2 macrophages. Further studies are needed to determine the balance between M1 and M2 macrophage polarization under the influence of FTDCs exposed to the inflammatory milieu of preeclampsia.

\section{References}

1. Klauber N, Rohan RM, Flynn E, D'Amato RJ: Critical components of the female reproductive pathway are suppressed by the angiogenesis inhibitor AGM-1470. Nat Med 1997, 3:443-446

2. Kaufmann P, Black S, Huppertz B: Endovascular trophoblast invasion: implications for the pathogenesis of intrauterine growth retardation and preeclampsia. Biol Reprod 2003, 69:1-7

3. Moffett-King A: Natural killer cells and pregnancy. Nat Rev Immunol 2002, 2:656-663

4. Barreda DR, Hanington PC, Belosevic M: Regulation of myeloid development and function by colony stimulating factors. Dev Comp Immunol 2004, 28:509-554

5. Yong K, Salooja N, Donahue RE, Hegde U, Linch DC: Human macrophage colony-stimulating factor levels are elevated in pregnancy and in immune thrombocytopenia. Blood 1992, 80:2897-2902

6. Hayashi M, Shibazaki M, Sohma R, Ohkura T, Inaba N: Serum levels of macrophage colony-stimulating factor after cesarean section, vaginal delivery, or laparotomy in normal pregnant women and gynecologic patients. Acta Obstet Gynecol Scand 2003, 82:597-602

7. Renaud SJ, Graham CH: The role of macrophages in utero-placental interactions during normal and pathological pregnancy. Immunol Invest 2008, 37:535-564

8. Huang SJ, Chen CP, Schatz F, Rahman M, Abrahams VM, Lockwood CJ: Pre-eclampsia is associated with dendritic cell recruitment into the uterine decidua. J Pathol 2008, 214:328-336

9. Huang SJ, Zenclussen AC, Chen CP, Basar M, Yang H, Arcuri F, Li M, Kocamaz E, Buchwalder L, Rahman M, Kayisli U, Schatz F, Toti P, Lockwood CJ: The implication of aberrant GM-CSF expression in decidual cells in the pathogenesis of preeclampsia. Am J Pathol 2010, 177:2472-2482 
10. Wu ZM, Yang H, Li M, Yeh CC, Schatz F, Lockwood CJ, Di W, Huang SJ: Pro-inflammatory cytokine-stimulated first trimester decidual cells enhance macrophage-induced apoptosis of extravillous trophoblasts. Placenta 2012, 33:188-194

11. Huang SJ, Schatz F, Masch R, Rahman M, Buchwalder L, Niven-Fairchild T, Tang C, Abrahams VM, Krikun G, Lockwood CJ: Regulation of chemokine production in response to pro-inflammatory cytokines in first trimester decidual cells. J Reprod Immunol 2006, 72 : 60-73

12. Smith SD, Dunk CE, Aplin JD, Harris LK, Jones RL: Evidence for immune cell involvement in decidual spiral arteriole remodeling in early human pregnancy. Am J Pathol 2009, 174:1959-1971

13. Hempstock J, Jauniaux E, Greenwold N, Burton GJ: The contribution of placental oxidative stress to early pregnancy failure. Hum Pathol 2003, 34:1265-1275

14. Allaire AD, Ballenger KA, Wells SR, McMahon MJ, Lessey BA: Placental apoptosis in preeclampsia. Obstet Gynecol 2000, 96: 271-276

15. Heazell AE, Buttle HR, Baker PN, Crocker IP: Altered expression of regulators of caspase activity within trophoblast of normal pregnancies and pregnancies complicated by preeclampsia. Reprod Sci 2008, 15: $1034-1043$

16. Levy R, Smith SD, Yusuf K, Huettner PC, Kraus FT, Sadovsky Y, Nelson DM: Trophoblast apoptosis from pregnancies complicated by fetal growth restriction is associated with enhanced p53 expression. Am J Obstet Gynecol 2002, 186:1056-1061

17. Chiu PM, Ngan YS, Khoo US, Cheung AN: Apoptotic activity in gestational trophoblastic disease correlates with clinical outcome: assessment by the caspase-related M30 CytoDeath antibody. Histopathology 2001, 38:243-249

18. Brown GC, Neher JJ: Eaten alive! cell death by primary phagocytosis: "phagoptosis". Trends Biochem Sci 2012, 37:325-332

19. Kinchen JM, Ravichandran KS: Phagocytic signaling: you can touch, but you can't eat. Curr Biol 2008, 18:R521-R524

20. Li M, Wu ZM, Yang H, Huang SJ: NFkappaB and JNK/MAPK activation mediates the production of major macrophage- or dendritic cell-recruiting chemokine in human first trimester decidual cells in response to proinflammatory stimuli. J Clin Endocrinol Metab 2011, 96:2502-2511

21. Guenther S, Vrekoussis T, Heublein S, Bayer B, Anz D, Knabl J, Navrozoglou I, Dian D, Friese K, Makrigiannakis A, Jeschke U: Decidual macrophages are significantly increased in spontaneous miscarriages and over-express FasL: a potential role for macrophages in trophoblast apoptosis. Int J Mol Sci 2012, 13:9069-9080

22. Haeger M, Unander M, Norder-Hansson B, Tylman M, Bengtsson A: Complement, neutrophil, and macrophage activation in women with severe preeclampsia and the syndrome of hemolysis, elevated liver enzymes, and low platelet count. Obstet Gynecol 1992, 79:19-26

23. Duclos AJ, Pomerantz DK, Baines MG: Relationship between decidual leukocyte infiltration and spontaneous abortion in a murine model of early fetal resorption. Cell Immunol 1994, 159:184-193

24. Jenkins SJ, Ruckerl D, Cook PC, Jones LH, Finkelman FD, van Rooijen N, MacDonald AS, Allen JE: Local macrophage proliferation, rather than recruitment from the blood, is a signature of TH2 inflammation. Science 2011, 332:1284-1288

25. Svensson J, Jenmalm MC, Matussek A, Geffers R, Berg G, Ernerudh J: Macrophages at the fetal-maternal interface express markers of alternative activation and are induced by M-CSF and IL-10. J Immunol 2011, 187:3671-3682

26. Hamilton TA, Zhao C, Pavicic PG Jr, Datta S: Myeloid colonystimulating factors as regulators of macrophage polarization. Front Immunol 2014, 5:554

27. Houser BL, Tilburgs T, Hill J, Nicotra ML, Strominger JL: Two unique human decidual macrophage populations. J Immunol 2011, 186:2633-2642

28. Reinartz S, Schumann T, Finkernagel F, Wortmann A, Jansen JM, Meissner W, Krause M, Schworer AM, Wagner U, MullerBrusselbach S, Muller R: Mixed-polarization phenotype of ascitesassociated macrophages in human ovarian carcinoma: correlation of CD163 expression, cytokine levels and early relapse. Int J Cancer 2014, 134:32-42

29. Boorsma CE, Draijer C, Melgert BN: Macrophage heterogeneity in respiratory diseases. Mediators Inflamm 2013, 2013:19

30. Yeh CC, Chao KC, Huang SJ: Innate immunity, decidual cells, and preeclampsia. Reprod Sci 2013, 20:339-353

31. Barclay AN: Signal regulatory protein alpha (SIRPalpha)/CD47 interaction and function. Curr Opin Immunol 2009, 21:47-52

32. Aldrich M, Sanders D, Lapteva N, Huang XF, Chen SY: SOCS1 downregulation in dendritic cells promotes memory T-cell responses. Vaccine 2008, 26:1128-1135

33. DiFederico E, Genbacev O, Fisher SJ: Preeclampsia is associated with widespread apoptosis of placental cytotrophoblasts within the uterine wall. Am J Pathol 1999, 155:293-301 